\title{
The Influence of Doppler on Spread Spectrum Signal Synchronization
}

\section{Yuming Zhu}

China Satellite Maritime Tracking and Control Department, Jiangyin, 214431, China

Keywords: spectrum; Doppler; synchronization.

\begin{abstract}
In this paper, the composition and principle of the existing spread spectrum measurement and control system are analyzed, and the characteristics of the signal Doppler shift and noise are discussed. The general concept and theoretical basis of the spread spectrum signal processing are established.
\end{abstract}

\section{Introduction}

Spread-spectrum unified monitoring and control system mainly by the ground system and space system composed of two parts. These two parts are composed of antenna, tracking servo, high power amplifier, up and down frequency converter, digital integrated baseband terminal equipment, spread spectrum measurement and control system based on Spread Spectrum Communication basic principle, mainly want to send information and a group of PN Code multiplication, so the spectrum has been extended, the receiver inverse operation, restore the original information data.

\section{Spectrum Analysis}

In the ideal transmission environment, the degree of signal distortion is mainly determined by the Gaussian noise and Doppler. The signal received by the receiver can be reduced to the following model:

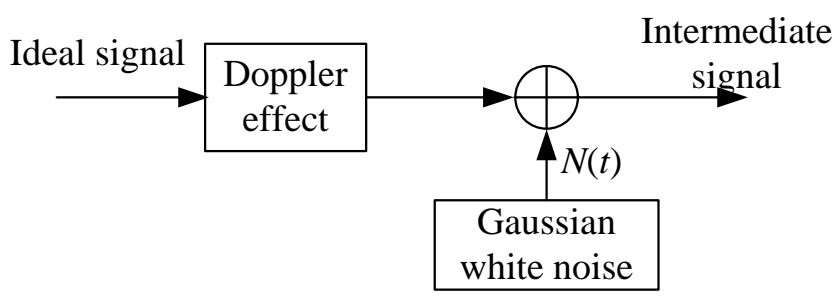

Figure 1. Receive signal simplification model

The signal obtained by the signal simplified model shown in Figure 1 can be expressed as:

$$
\begin{aligned}
s(t)= & \sqrt{2 P_{I}} \cdot P N_{I}(t) \cdot d(t) \cdot \cos \left(\omega_{0} t+\omega_{d}(t) t+\varphi(t)\right) I_{N}(t) \\
& +\sqrt{2 P_{Q}} \cdot P N_{Q}(t) \cdot \sin \left(\omega_{0} t+\omega_{d}(t) t+\varphi(t)\right) Q_{N}(t)
\end{aligned}
$$

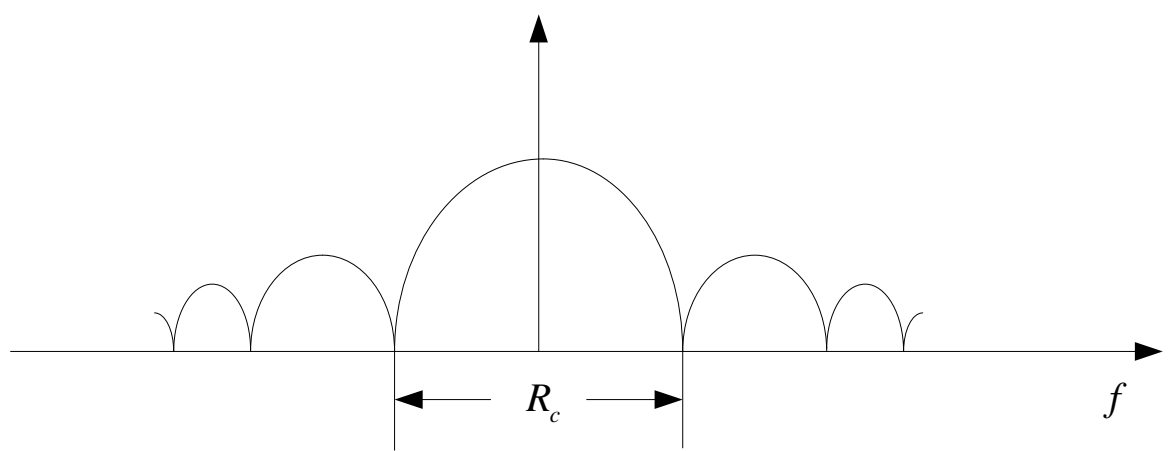

Figure 2. Spread Spectrum Measurement and Control Signal Theoretical Spectrum

QPSK modulation of the spread spectrum measurement and control signal theoretical spectrum shown in Figure 2, the signal bandwidth is equal to the signal rate $R_{c}$. Figure 3 is the spread spectrum measurement and control signal source generated $70 \mathrm{MHz}$ IF signal with $6.5 \mathrm{MHz}$ under-sampling 
signal spectrum. Figure (a) is the noise-free signal spectrum. Figure (b) is the Doppler frequency and noise signal spectrum, signal to noise ratio of 0dB, Doppler frequency offset $100 \mathrm{kHz}$, we can see that the signal to noise ratio is low, the signal is almost submerged in the noise.

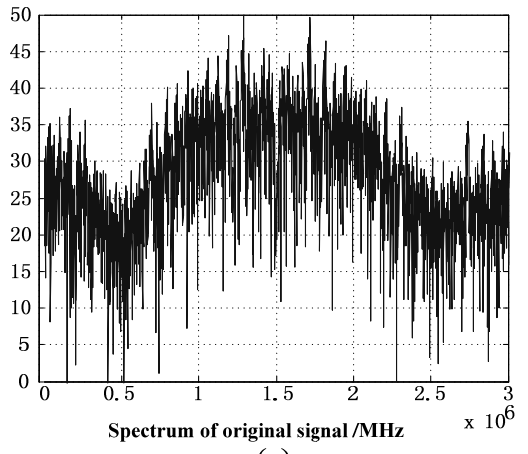

(a)

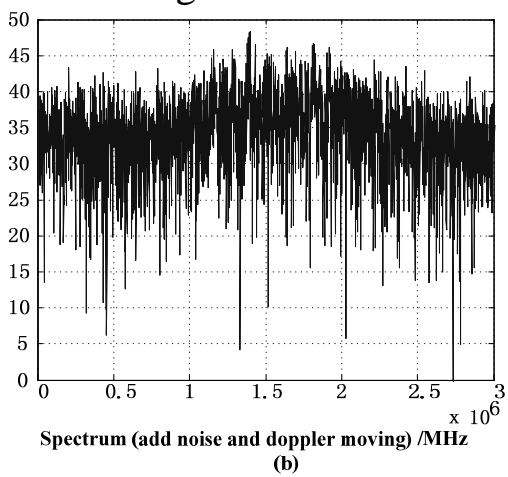

Figure 3. Spectral comparison chart of spread spectrum measurement and control signal

Pseudo-code synchronization process down-converter, coherent accumulator for the linear system. And the signal and noise are independent of each other, the influence of the Doppler effect on the useful signal and noise can be considered separately. The following analysis of the Doppler effect on the synchronization process. The Doppler effect of the received signal depends on the motion trajectory and velocity variation of the measurement and control target. The Doppler effect has an effect on the carrier frequency and the pseudo code, which increases the uncertainty of the synchronization process. Spread spectrum signal synchronization process is generally achieved through the code correlation, code delay; Doppler frequency shift will lead to deterioration of related performance. Since the effects of the two on the cumulative coupling are very small, almost negligible. Thus, the effect of the carrier Doppler effect and the code-frequency Doppler effect on the synchronization process will be discussed below.

\section{Influence of Carrier Doppler Shift on Synchronization Algorithm}

In the telemetry remote control process, when there is relative movement between the monitoring station and the monitoring and control target, the received signal carrier frequency will occur frequency offset. Whether the transmitter movement or the receiver movement, as long as the transmitter and receiver radial relative velocity of $\mathrm{V}$, the receiving frequency becomes:

$$
f_{r}=\left(1 \pm \frac{v}{c}\right) f_{b}
$$

In general, the frequency of the transmitter and the receiver frequency to do poor, that is, Doppler:

$$
f_{d}=f_{r}-f_{b}= \pm \frac{v}{c} f_{b}
$$

From the formula (2) can be seen, the higher the signal frequency, the shorter the wavelength. The faster the relative motion, the greater the Doppler of the received signal. Taking into account the Doppler factor, the receiver can receive the signal can be expressed as:

$$
s(t)=\sqrt{2 P} P N(t) \cos \left[2 \pi\left(f_{b}+f_{d}\right) t+\varphi\right]
$$

The effect of carrier frequency Doppler on the capture of pseudo codes is independent of the sampling rate. After sampling the same phase, the orthogonal volume is:

$$
\begin{aligned}
& I(k)=\sqrt{2 P} P N(k) \cos \left(2 \pi f_{d} T_{s} k+\varphi\right) \\
& Q(k)=-\sqrt{2 P} P N(k) \sin \left(2 \pi f_{d} T_{s} k+\varphi\right)
\end{aligned}
$$

The relevant cumulative results are:

$$
\sqrt{\sum^{2} I+\sum^{2} Q}=\sqrt{2 P} \cdot R_{P N}(\tau) \cdot\left|\frac{\sin \left(\pi \cdot f_{d} \cdot T\right)}{N \sin \left(\pi \cdot f_{d} \cdot T_{s}\right)}\right|
$$


It can be seen from the formula (7) that the influence of the Doppler frequency on the correlation cumulative result is that the correlation peak is reduced by $\left|\frac{\sin \left(\pi \cdot f_{d} \cdot T\right)}{N \sin \left(\pi \cdot f_{d} \cdot T_{c}\right)}\right|$.

\section{Influence of Frequency - frequency Doppler on Synchronization Algorithm}

As the Doppler will cause the transmitter and receiver frequency reference is different, so the receiver and the transmitter PN code frequency is different, so the PN code width is different, which will affect the PN code synchronization.

When there is a relative movement speed between the transmitter and the receiver, the reception code chip width and the transmission code chip width have the following relationship:

$$
T_{r c}=\frac{1}{1 \pm \frac{v}{c}} T_{s c}
$$

Define the normalized PN code The ratio of the difference between the frequency reference of the received PN code and the frequency reference of the transmitted PN code to the transmitted code clock frequency, ie,

$$
\varepsilon=\frac{f_{r c}-f_{s c}}{f_{s c}}=\frac{\frac{1}{T_{r c}}-\frac{1}{T_{s c}}}{\frac{1}{T_{s c}}}= \pm \frac{v}{c}
$$

There is the following relationship between the received code and the chip width of the transmit code:

$$
T_{r c}=\frac{1}{(1+\varepsilon)} T_{s c} \approx(1-\varepsilon) T_{s c} \quad(\because \varepsilon<<1)
$$

Since the PN code synchronization process is fast, it can be considered in the process of synchronization Doppler for a constant value, while the requirements of this time within the Doppler code relative to sliding less than a code.

The phase relationship between the local code and the received code is shown in Fig.

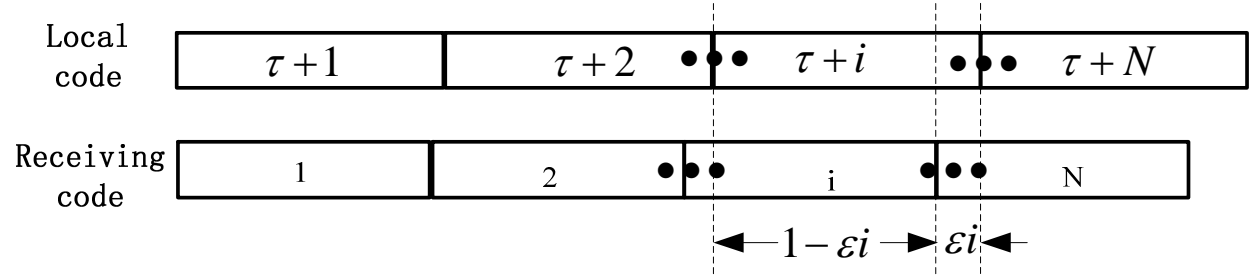

Figure 4. The phase relationship between the local code and the received code $(\varepsilon>0)$ The cumulative result is:

$$
\begin{aligned}
y(\varepsilon, \tau) & =\sum_{i=1}^{N}\left[(1-\varepsilon i) P N_{i} P N_{\tau+i}+(\varepsilon i) P N_{\tau+i} P N_{i+1}\right] \\
& =\left\{\begin{array}{cl}
N\left[1-\frac{1}{2}(N+1) \varepsilon\right] & , \tau=0 \\
0 & , \tau=-1 \\
\frac{1}{2} N(N+1) \varepsilon & , \tau=+1
\end{array}\right.
\end{aligned}
$$

When the delay is less than zero, then the local code and receive code phase relationship shown in Figure 5. 


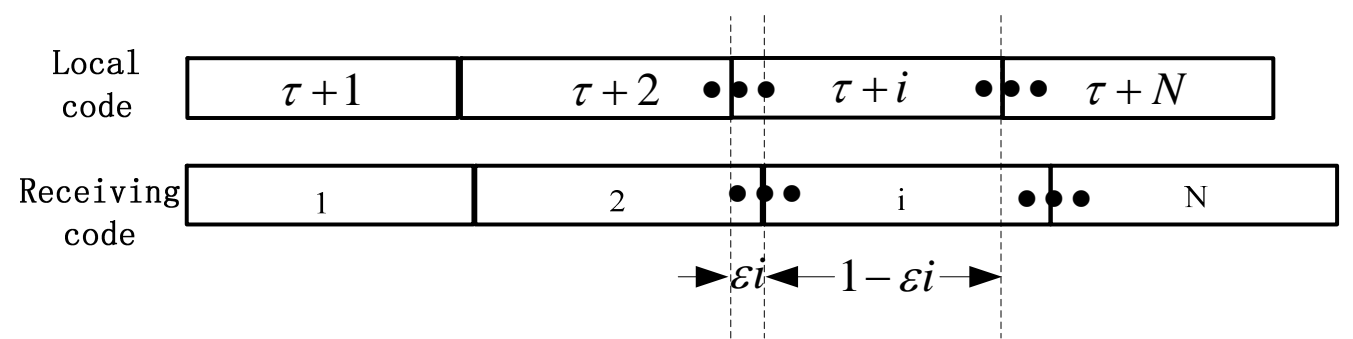

Figure 5. The phase relationship between the local code and the received code $(\varepsilon<0)$ The cumulative result is:

$$
\begin{aligned}
& y(\varepsilon, \tau)=\sum_{i=1}^{N}(-\varepsilon i) P N_{i-1} P N_{\tau+i}+\sum_{i=1}^{N}(1+\varepsilon i) P N_{i} P N_{\tau+i} \\
& =\left\{\begin{array}{cl}
N\left[1+\frac{1}{2}(N+1) \varepsilon\right] & , \tau=0 \\
-\frac{1}{2} N(N+1) \varepsilon & , \tau=-1 \\
0 & , \tau=+1
\end{array}\right.
\end{aligned}
$$

According to the formula (11) and the formula (12), it can be seen that the value of the correlation peak between the receiver and the transmitter is not the maximum when $\varepsilon>0$ and $\varepsilon<0$. When $|N \varepsilon| \rightarrow 1$, Doppler will cause the side lobe peak and the peak of the main lobe is very close, so the probability of false alarm will be great. Under normal circumstances, the relative speed of the aircraft is relatively small, short code unit width is large, so generally do not care about the impact of the multi-Pratt to code.

\section{Summary}

In this paper, the basic principle and system composition of spread spectrum signal synchronization are briefly summarized. The general aspects of the spread spectrum measurement and control signal structure, spread code and Doppler frequency shift and noise synchronization are briefly introduced.

\section{References}

[1]. John J.Schamus, Veridian Engineering, Dayton OH.Real-Time Software GPS Receiver [J].ION GPS 2012, 24-27.

[2]. Grydeland T, Lind F.D, Erickson P.J, etal. Software Radar Signal Processing[J].European Geosciences Union 2014.

[3]. A.Papoulis, Probability.Random Variables and Stochastic Processes [M].MeGraw-Hill.New York.2004. 